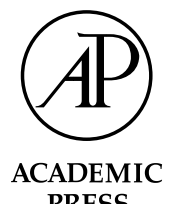

\title{
Brain imaging of tongue-twister sentence comprehension: Twisting the tongue and the brain
}

\author{
Timothy A. Keller, Patricia A. Carpenter, \\ and Marcel Adam Just* \\ Center for Cognitive Brain Imaging, Carnegie Mellon University, Pittsburgh, PA 15213, USA
}

Accepted 14 May 2002

\begin{abstract}
This study used fMRI to investigate the neural basis of the tongue-twister effect in a sentence comprehension task. Participants silently read sentences equated for the syntactic structure and the lexical frequency of the constituent words, but differing in the proportion of words that shared similar initial phonemes. The manipulation affected not only the reading times and comprehension performance, but also the amount of activation seen in a number of language-related cortical areas. The effect was not restricted to cortical areas known to be involved in articulatory speech programming or rehearsal processes (the inferior frontal gyrus and anterior insula), but also extended to areas associated with other aspects of language processing (inferior parietal cortex) associated with phonological processing and storage. (C) 2002 Elsevier Science (USA). All rights reserved.
\end{abstract}

Keywords: Sentence comprehension; Brain imaging; fMRI; Phonological processes; Working memory

\section{Introduction}

The role of phonological processing in silent reading has been intensely debated without respite for decades. Although there is little doubt that phonological representations are important in learning to read (see e.g., Gathercole \& Baddeley, 1993; Johnston, Thompson, Gletcher-Flinn, \& Holligan, 1995; Wagner \& Torgeson, 1987), the role of phonological processing in the skilled reading of adults remains an area of controversy. Some researchers report evidence of prelexical activation of phonological information (e.g., Lukatela \& Turvey, 1990, 1994a, 1994b; Naish, 1980; Van Orden, 1987; Van Orden, Pennington, \& Stoner, 1990), while others find no such effects (e.g., Seidenberg, Waters, Barnes, \& Tanenhaus, 1984; Waters \& Seidenberg, 1985; Waters, Seidenberg, \& Bruck, 1984). Nevertheless, a role for phonologically mediated access to semantics in skilled readers is accepted by both dual-route (e.g.,

${ }^{*}$ Corresponding author. Fax: 1-412-268-2804.

E-mail address: just+@cmu.edu (M.A. Just). 
Coltheart, Brent, Atkins, \& Haller, 1993) and connectionist (e.g., Plaut, McClelland, Seidenberg, \& Patterson, 1996; Seidenberg \& McClelland, 1989) models of lexical access, although such models differ in terms of the situations, timing, and degree to which such processes play a role. The current study brings the technique of functional brain imaging to bear on the question of which psychological processes related to phonology are affected during the silent reading of tongue-twister sentences.

The role of phonological processing in reading has usually been studied in the context of recognizing single words rather than in the context of sentence comprehension. One sentence paradigm that has been used to demonstrate such a role involves the comparison of silent reading of "tongue-twisters," sentences which contain a large proportion of words that share the same initial phonemes, with those that do not. Such sentences produce slower reading times for skilled readers, whether the reading is silent or overt (Haber \& Haber, 1982). This tongue-twister effect on reading times is also found for the reading of extended texts for comprehension in both English (Ayres, 1984) and in logographic writing systems such as Chinese (Zhang \& Perfetti, 1993). Skilled readers are also slower and less accurate in verifying the semantic acceptability of sentences that contain a large proportion of words that share the same initial phoneme or that contain a mixture of words whose initial phonemes share the same place of articulation (e.g. / b/ and /p/), but which differ in voicing (McCutchen, Bell, France, \& Perfetti, 1991; McCutchen \& Perfetti, 1982). In addition, congenitally deaf readers show comparable effects in semantic acceptability judgments (Hanson, Goodell, \& Perfetti, 1991), suggesting that the role of phonology in reading is not simply a residual effect of learning to read with the support of auditory input. In summary, the slowing of the reading process by phonological similarity suggests that phonological or articulatory codes are being activated in normal reading.

Two main possible accounts of the tongue-twister effect have been suggested. One account, articulatory based, is that the tongue-twister effect results from a bottleneck in subvocal phonological/articulatory processing at a prelexical stage or in lexical access (Haber \& Haber, 1982). Such an articulatory locus could produce a deficit in comprehension or memory maintenance by diverting cognitive resources from comprehension or maintenance processes to the processing of phonological/ articulatory codes or to the repair of phonological errors. The second account, memory-based, is that the difficulty of tongue-twister sentences results because the phonological similarities interfere with the normal maintenance of the surface structure of the sentence within a verbal working memory system (e.g., the articulatory or phonological loop of Baddeley \& Hitch, 1974) during syntactic and semantic comprehension. Such interference might require reprocessing of the words in order to obtain more complete information about the words (McCutchen \& Perfetti, 1982) or about their order in the sentence (McCutchen et al., 1991), adding to the processing time. Both accounts enjoy empirical support, and they are not mutually exclusive.

The evidence to support the articulatory account is not unequivocal. Attempts to provide evidence of specific articulatory interference between reading tongue-twister sentences and secondary tasks involving the same articulatory features in wordinitial phonemes have been mixed. When participants overtly articulated phrases involving repetition of the same place of articulation of the initial consonants concurrently with silently reading tongue-twister sentences for a semantic acceptability judgment, there was no evidence of an interaction between the place of articulation for the phrase and for the sentence (McCutchen \& Perfetti, 1982). On the other hand, phoneme-specific interference occurred when participants silently maintained a sequence of numbers that shared the same place of articulation as tongue-twister sentences that were simultaneously read (McCutchen et al., 1991). In this latter 
study, latencies and errors increased for both the primary acceptability-judgment task and a secondary number-recall task.

The evidence to support the account that the tongue-twister effect is restricted to a post-lexical, phonological memory process is also problematic. If phonological processing contributes to lexical access, the interference caused by phonological similarity in tongue-twisters could have its effect early in processing at a pre-lexical stage or during lexical access. On the other hand, both the visual and phonological similarity in tongue-twisters might lead one to expect a priming effect on lexical access. McCutchen et al. (1991) found no tongue-twister effect when participants made lexical decisions on lists of words that either began with the same phoneme or did not, suggesting that phonological similarity does not interfere with lexical access. Furthermore, the phoneme-specific interference between silently maintained numbers and tongue-twister sentences in the acceptability judgment task discussed above was not found in the lexical-decision task. Robinson and Katayama (1997), however, demonstrated a tongue-twister effect on reaction times in a lexical decision task using slightly longer lists of words, and perhaps more importantly, tongue-twister lists that alternated initial phonemes or phoneme clusters (e.g., flagpole, fractional, flopping, and fragrant), rather than simply those composed of words with the same initial consonant. This alternation of initial phoneme clusters may have reduced similarity effects in orthographic or phonological priming, and it is known to be particularly effective in slowing overt speech production (Rosenbaum, Weber, Hazelett, \& Hindorff, 1986), and inducing overt speech errors involving perseverations or exchanges. Because the frequency and types of speech errors are similar for overt and covert production tasks (e.g., Postma \& Noordanus, 1996), the Robinson and Katayama (1997) stimuli may have been more potent tongue-twisters, allowing the effect to be seen in a lexical decision task.

A phenomenon that appears to be related to the tongue-twister effect occurs in the serial recall of a list of phonologically similar verbal items, where recall is poorer than with phonologically dissimilar items (e.g., Baddeley \& Lewis, 1981; Conrad, 1964). This phonological similarity effect has traditionally been investigated by manipulating the vowels rather than the initial phonemes of words in the input list (e.g., pat, man, cat, and hand). However, the same deficit in recall has also recently been found for lists containing words with the same initial consonant relative to lists varying in initial consonants (Robinson \& Katayama, 1997), increasing the resemblance to the tongue-twister effect. Just as there are two accounts of the tongue-twister effect, there are two approximately corresponding accounts of the phonological similarity deficit in serial recall found by Robinson and Katayama (1997). One possible explanation of the phonological similarity deficit in serial recall is that it is due to phonological confusions within a temporary memory system. An alternative account is that it is due to it taking longer to covertly articulate lists containing phonologically similar initial consonants, a factor known to decrease serial recall (Baddeley, Thomson, \& Buchanan, 1975). It may be that the issues concerning phonological similarity and the answers are the same in the tongue-twister and serial recall paradigms.

Findings from functional neuroimaging are providing a new perspective for framing such questions about tongue-twisters and phonological processes. Covert articulatory processes have been associated with activation in left inferior frontal gyrus (roughly Broca's area), left middle frontal gyrus, and in the supplementary motor area in a number or neuroimaging studies using both PET (Demonet et al., 1992; Fiez et al., 1996; Frith, Friston, Liddle, \& Frackowiak, 1991; Jonides et al., 1998; Paulesu, Frith, \& Frackowiak, 1993; Wise et al., 1991) and fMRI (Chee, Tan, \& Thiel, 1999; Cuenod et al., 1995; Henson, Burgess, \& Frith, 2000; Hinke et al., 
1993). These same areas are activated in tasks requiring memory for visually presented verbal information, presumably because such tasks require subvocal articulatory recoding and rehearsal processes. In addition to frontal activation, however, verbal working memory tasks also result in activation in left or bilateral parietal cortex (Awh et al., 1996; Henson et al., 2000; Honey, Bullmore, \& Sharma, 2000; Jonides et al., 1998; Paulesu et al., 1993), or in posterior superior temporal cortex (Fiez et al., 1996), leading to the suggestion that these areas function as a site of phonological storage. Neuropsychological evidence for an anatomical dissociation between the phonological store and the articulatory control process has also been relatively consistent in suggesting that patients with damage to the supramarginal, angular, and superior and middle temporal gyri show behavioral effects consistent with an impaired phonological store (Vallar, Di Betta, \& Silveri, 1997; Warrington, Logue, \& Pratt, 1971), while those showing patterns consistent with an intact phonological store and impaired articulatory rehearsal processes have a sparing of these areas combined with damage to inferior frontal premotor and insula regions, and to cerebellar regions (Vallar et al., 1997; Vallar \& Cappa, 1987).

Functional neuroimaging results also indicate that a large-scale network of brain areas is recruited in the service of sentence comprehension, and that the network dynamically adapts itself to the computational demands of the particular types of comprehension items that are encountered (Just, Carpenter, Keller, Eddy, \& Thulborn, 1996). Moreover, the effect of a particular type of additional workload (such as additional syntactic or lexical processing) is propagated to several areas, indicating a high degree of interaction among the areas (Keller, Carpenter, \& Just, 2001). These new perspectives predict that the effect of a tongue-twister sentence is not likely to be isolated to a single cortical area. Instead, a significant additional workload is likely to impact not only the area that provides the primary substrate for the relevant processing, but is also likely to impact the other network members that interact or collaborate with that area. The cortical network for language processing is a complex system and the additional processing difficulty engendered by a tongue-twister sentence is likely to be manifested in multiple areas that underlie the processing of sentence comprehension. Thus the opposition between the two accounts of the tongue-twister effect may be a false dichotomy. The possible reason for a lack of empirical support for a single mechanism underlying the tongue-twister effect may be provided by this perspective.

The present study examined the tongue-twister effect in sentence comprehension by using functional magnetic resonance imaging (fMRI) to quantify the amount of activation in cortical areas known to be involved in language processing. We compared the amount of fMRI-measured activation (relative to a common baseline) in a task where participants silently read tongue-twister sentences (e.g., Several short Swedish sword swallowers shifted some swords swiftly.), versus control sentences (e.g., Seven tall German circus performers juggled many knives quickly.). The tongue-twister sentences contained a large proportion of words that shared the initial letter and phoneme, as in previous behavioral work on comprehension of tongue-twister sentences (Ayres, 1984; McCutchen et al., 1991; McCutchen \& Perfetti, 1982; McCutchen, Dibble, \& Blount, 1994; Zhang \& Perfetti, 1993). However, the words also alternated in terms of initial phoneme clusters, as in the Robinson and Katayama (1997) study of tongue-twister effects in lexical decisions and memory span.

In contrast to most previous behavioral studies of the tongue-twister effect in reading comprehension, which have used a semantic acceptability-judgment task, the present study explicitly probed participants' comprehension by requiring them to answer a true-false question about the content of each sentence. For example, a tongue-twister sentence was Five fashionable females flew to France for fresh fashions, 
followed by the probe that was to be verified as true or false: Five females flew to France? A control sentence for that stimulus, which would be read by a different participant, would be Seven energetic women flew to Dallas for lively parties, and the probe for it was: Seven women flew to Dallas? An example of a tongue-twister followed by a false probe is $A$ woman in a simple shawl shoveled soft snow slowly. Probe: A man in a shawl shovelled snow?

The goal of the study was to identify cortical areas within the language-processing network that increase in activation in response to tongue-twister sentences. It is well established across a variety of domains and cognitive tasks, that increases in task difficulty can be related to increases in fMRI-measured blood-oxygenation level dependent (BOLD) signal intensity (e.g., Braver, Cohen, Jonides, Smith, \& Noll, 1997; Carpenter, Just, Keller, Eddy, \& Thulborn, 1999; Grasby et al., 1994; Just et al., 1996; Rypma, Prabhakaran, Desmond, Glover, \& Gabrieli, 1999). Finding differences among regions in the response to phonological similarity may provide insight into the locus or loci of the tongue-twister effect in silent reading. For instance, to the extent that the tongue-twister results from a bottleneck in subvocal phonological or articulatory processing, one would expect an effect of phonological similarity in the left frontal lobe speech motor and premotor areas, centrally including Broca's area. To the extent that the tongue-twisters place additional demands on verbal working memory or other phonological processes involved in reading, one would expect increases in activation in a more widely distributed network of cortical areas, involving not only Broca's area, but also the inferior parietal area implicated in phonological storage, and the posterior left superior/middle temporal area implicated in lexical, semantic, and syntactic processing.

\section{Methods}

\subsection{Participants}

The participants were 15 right-handed college students at Carnegie Mellon University (eight female, seven male). Data from two additional participants were discarded because of excessive estimated head motion during the functional imaging (see below). The mean age of the remaining participants was 20.9 years $(\mathrm{SD}=2.2$ years). The mean handedness score, assessed by the Edinburgh Handedness Inventory (Oldfield, 1971), was $71.8(\mathrm{SD}=16.4)$. Each participant gave signed informed consent that had been approved by the University of Pittsburgh and the Carnegie Mellon Institutional Review Boards. Prior to being tested in the scanner, each participant was familiarized with the task, the scanner and the fMRI procedure. During a practice session, participants were presented with 10 trials each of tonguetwister and control sentences to ensure that they understood the task.

\subsection{Procedure and experimental paradigm}

The experiment consisted of two experimental conditions and one control condition. Tongue-twister sentences contained a high proportion of words that began with the same letter, although the words varied the initial phoneme cluster. For each tongue-twister sentence, there was a yoked control sentence of the same syntactic structure but with words that did not begin with the same letter. The total length of the sentences in characters was equated across conditions, and the mean lexical frequency of the content words in the sentences was roughly equivalent according to the Kucera and Francis (1967) norms. 
The sentences were projected onto a translucent plastic screen attached to the roof of the bore of the scanner. Participants viewed the screen through a pair of mirrors at a total distance of approximately $20 \mathrm{~cm}$. At this distance, a single line of the sentence stimuli subtended a visual angle of approximately $30^{\circ}$. A sentence and a true/false probe question were presented on the screen simultaneously until the participant responded. Participants were instructed to first silently read the sentence at their own pace, then to read the probe question, and finally to signal their answer with a button press. The questions matched the sentence type in that they either included similar initial phonemes or did not. Within each condition, $60 \%$ of the questions were true and $40 \%$ were false. Tongue-twister and control sentences were presented in separate epochs consisting of five sentences each. Between each epoch, a 6-s rest was presented to allow the hemodynamic response to return to a resting state. In addition, after every two epochs of experimental conditions, a 24-s fixation interval during which participants were asked to fixate an asterisk was presented, to obtain a control baseline measure of brain activation with which to compare experimental conditions. There were four epochs of each of the experimental conditions in addition to six fixation epochs. The experimental epochs and fixation epochs were presented in a pseudo-randomized order to control for practice effects.

\section{3. $f M R I$ acquisition parameters}

The imaging was carried out at the University of Pittsburgh Magnetic Resonance Research Center on a 3.0 T General Electric Signa scanner (Thulborn et al., 1996). The images were acquired with a G.E. quadrature birdcage head coil. For the functional imaging a $T_{2}{ }^{*}$-weighted gradient echo EPI pulse sequence was used with $\mathrm{TR}=3000 \mathrm{~ms}, \mathrm{TE}=25 \mathrm{~ms}$, and a flip angle of $90^{\circ}$. Fourteen adjacent oblique-axial slices were acquired in an interleaved sequence, with $5 \mathrm{~mm}$ slice thickness, $1 \mathrm{~mm}$ slice gap, a $40 \mathrm{~cm} \times 20 \mathrm{~cm} \mathrm{FOV}$, and $128 \times 64$ matrix size, resulting in in-plane resolution of $3.125 \mathrm{~mm} \times 3.125 \mathrm{~mm}$. Two sets of structural images were acquired for each participant to be used in parcellating the functional images into anatomically predefined regions of interest. A 124-slice axial $T_{1}$-weighted $3 \mathrm{D}$ spoiled GRASS volume scan was acquired for each participant with $\mathrm{TR}=25 \mathrm{~ms}$, $\mathrm{TE}=4 \mathrm{~ms}$, flip angle $=40$, $\mathrm{FOV}=18 \mathrm{~cm}$, and a $256 \times 256$ matrix size, resulting in voxel dimensions of $0.9375 \mathrm{~mm} \times 0.9375 \mathrm{~mm} \times 1.5 \mathrm{~mm}$ thick. In addition, a 14-slice $T_{2}{ }^{*}$-weighted gradient echo EPI two-shot Instascan Multi-Echo Multi-Planar (ISMEMP) pulse sequence was acquired with the catch-and-hold option and with $\mathrm{TR}=10 \mathrm{~s}, \mathrm{TE}=112 \mathrm{~ms}$, flip angle $=90^{\circ}, \mathrm{FOV}=40 \times 20$, and a $512 \times 128$ matrix. The slice locations and thickness were the same as those for the functional single-shot EPI data; however this sequence provides an in-plane resolution of $0.78125 \mathrm{~mm} \times 1.5625 \mathrm{~mm}$ and shows susceptibility artifacts that are similar to those found in the functional acquisition sequence, facilitating co-registration.

\subsection{Definition of regions of interest}

To compare the amount of activation in a given area between conditions, anatomically defined ROIs were drawn for each participant using the parcellation scheme described by Rademacher, Galaburda, Kennedy, Filipek, and Caviness (1992) and further refined by Caviness, Meyer, Makris, and Kennedy (1996). This method uses limiting sulci and anatomically landmarked coronal planes to segment cortical regions. The ROIs were defined by a staff research assistant, after extensive training on the Rademacher/Caviness parcellation scheme. The anatomical information in the structural images was displayed in the three orthogonal planes 
simultaneously and the ROIs were manually drawn for each corresponding functional slice. Because the ROIs are defined on the basis of the structural images, and without reference to the functional activation, the method ensures that the location of activation does not bias the definitions of the ROIs. The inter-rater reliability of this ROI-defining procedure between two trained staff members was evaluated for four ROIs in two participants in another study. The reliability measure was obtained by dividing the size of the set of voxels that overlapped between the two raters by the mean of their two set sizes. The resulting eight reliability measures were in the 78 $91 \%$ range, with a mean of $84 \%$, as high as the reliability reported by the developers of the parcellation scheme.

In the Caviness et al. (1996) nomenclature, the inferior parietal ROI corresponded to inferior portions of the posterior supramarginal gyrus (SGp, BA 40) and the angular gyrus (AG, BA 39). The extrastriate occipital/inferior temporal ROI included the fusiform gyrus regions (TOF and TF, BA 36, 37, and 20) and the inferior temporal gyrus areas (TO3 and TFp or BA 37, and 20). The superior temporal ROI corresponds to the superior temporal gyrus (T1a and T1p or BA 22) and the middle temporal gyrus regions (T2a, T2p, and TO2 or BA 21, 22, and 37). The inferior frontal ROI consisted of the orbital, pars triangularis and pars opercularis portions of the inferior frontal gyrus, (FOC, F3t and F3O, BA 47, 45, and 44), and also included the frontal insula region (INS or BA 13).

The anatomical definition for the frontal eye fields (FEF) included the portion of the precentral sulcus that forms the posterior border of the middle frontal gyrus. This area corresponds approximately to the inferior portions of Brodmann's areas 6 within the precentral sulcus. The entire region of the middle frontal gyrus anterior to the FEF ROI was included in the dorsolateral prefrontal cortex (DLPFC) ROI and it encompasses Brodmann's areas 10, 9, 46, and portions of 8 and 6 .

\section{5. fMRI data analysis}

The image preprocessing corrected for signal drift and head motion (Eddy et al., 1995). The in-plane slicewise maximum estimated displacement (an average of translations in the $x$ and $y$ dimensions and rotations around the $z$ axis) of the 15 participants never exceeded 0.2 voxels, $(0.625 \mathrm{~mm})$ and the mean estimated displacement across participants was less than .01 voxels.

The data analysis focuses on quantifying BOLD-contrast-related changes in the fMRI-measured signal using a dependent measure which takes into account both the volume of activation and the percentage change in signal relative to a baseline level (Xiong, Rao, Gao, Woldorff, \& Fox, 1998). For each voxel in the a priori defined ROIs, the distribution of signal in an experimental condition was compared to that for the fixation condition using a $t$ test with a threshold of $t>6.0$. This threshold was selected to give a Bonferroni-corrected alpha level of .01 given approximately 5000 voxels per participant in all regions of interest. The integral of the percentage change in signal intensity was then calculated for each region in each condition by simply summing the percentage change in signal intensity relative to the baseline estimate across voxels that reached the critical threshold for that condition. Differences between conditions were then assessed for each of the regions by paired $t$ tests.

Because the experiment was participant-paced in order to allow online measurement of reading times and to provide a more realistic sentence-processing situation, longer sentence reading times as a function of task difficulty were expected. Thus task difficulty might be correlated with either the temporal dynamics of the BOLD response or the statistical power of the selection criteria across conditions. Because of these concerns, the analyses reported below were applied to the activation obtained in 
each condition after the condition durations were truncated to equal length across conditions on an individual participant basis. For each participant, the total amount of time spent in each of the two conditions was calculated from the behavioral data. The condition with the longer duration was then truncated by discarding images from the end of the longest block of that condition until this block was equal in length to the second longest block. If necessary, this procedure was repeated reiteratively (i.e., images were discarded from the end of the two longest blocks until they were equal in length to the third longest) until the total number of images in the two conditions was equivalent. This procedure ensured that no differential effect of trial duration could affect the means or variances of the activation across conditions. To be certain that this truncation procedure did not fundamentally alter the results, we also carried out all of the analyses using untruncated data, which produced identical conclusions, with one minor exception, which will be described below.

To reduce the possible influence of larger vessels, we excluded from analysis any voxels showing a large percentage change in signal intensity relative to the fixation condition (greater than $6.0 \%$ ). These constituted less than $1 \%$ of the voxels in all of the regions except the left inferior temporal/occipital extrastriate region. For this ROI, an average of $1.7 \%$ of the voxels per participant were excluded from the analysis. The larger number of voxels excluded in this region is consistent with its proximity to large draining veins along the tentorum cerebelli.

\section{Results}

\subsection{Behavioral data}

Participants spent significantly more time reading tongue-twister sentences and responding to the probe questions (mean $=7300 \mathrm{~ms}, \mathrm{SE}=476 \mathrm{~ms}$ ) than reading the control sentences and probes $(\mathrm{mean}=6172 \mathrm{~ms}, \mathrm{SE}=414 \mathrm{~ms}$ ), demonstrating a robust tongue-twister effect in silent sentence comprehension $[t(14)=9.55, p<.0001]$. Of equal interest, however, is the finding that participants also made more comprehension errors in the tongue-twister condition (mean $=9.6 \%, \mathrm{SE}=1.4 \%$ ) compared to the control condition (mean $=3.7 \%, \mathrm{SE}=1.1 \%$ ). This difference was also reliable $[t(14)=3.37, p<.005]$, indicating that the influence of phonological similarity in silent reading affects not only the rate of processing, but also the accuracy (see Fig. 1).

\subsection{Functional activation data}

Modulation of the amount of activation by phonological similarity. A number of the ROIs showed increases in activation for the tongue-twister condition compared to the control sentences, mirroring the behavioral results. The ROIs showing the increase were not restricted to the frontal speech motor and premotor areas, but also included inferior parietal areas. This more widespread result is consistent with a more distributed view of the sources of the effect, suggesting the involvement of the phonological-articulatory loop in the tongue-twister comprehension. To illustrate the results for a single participant, Fig. 2 shows the thresholded $t$-maps for two oblique-axial slices for each of the two conditions relative to fixation, and superimposed on the corresponding structural images; the voxels that are significantly more active than baseline are indicated in color. As indicated by the figure, the number of active voxels relative to the fixation baseline was greater in each of the left-hemisphere perisylvian language areas when this participant was performing the tongue-twister condition compared to the control condition. Fig. 3 plots the 

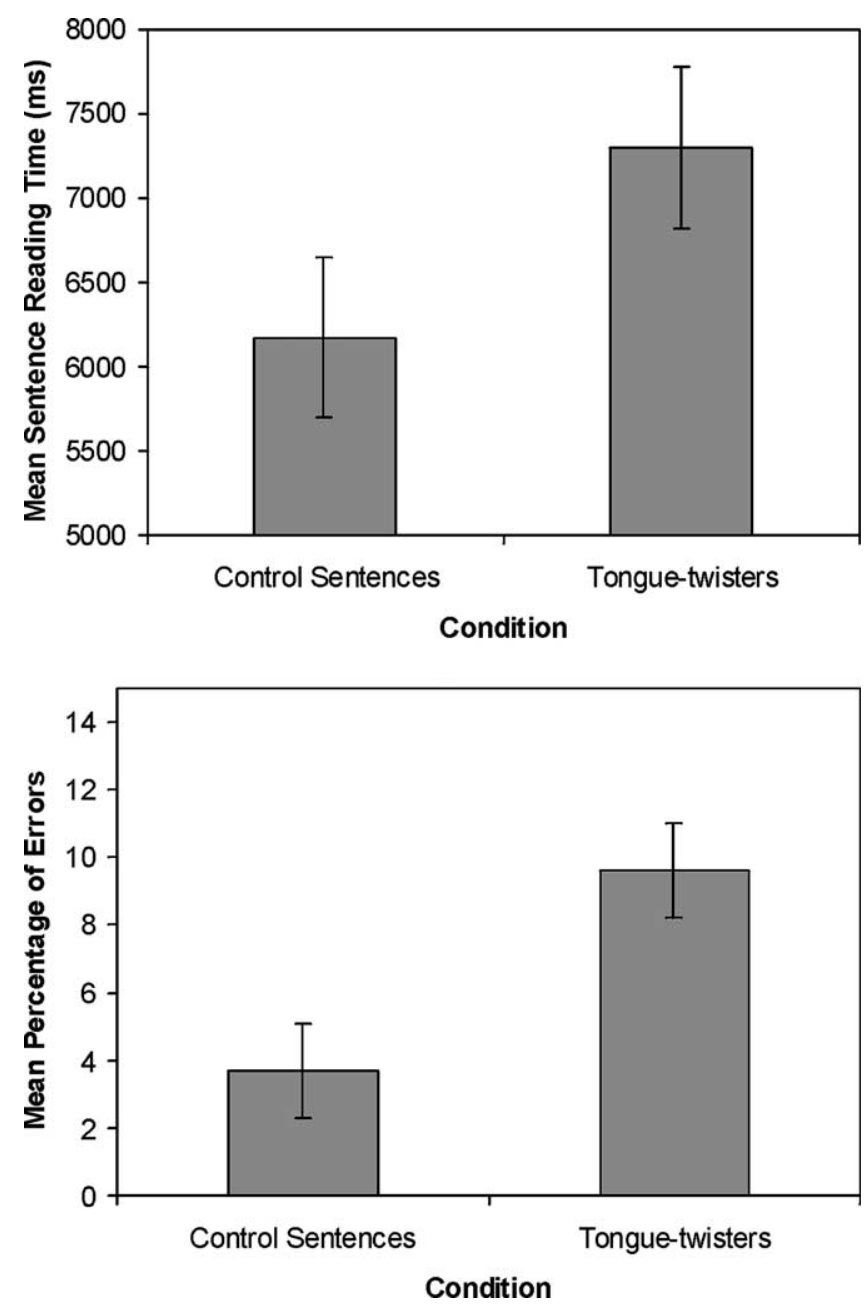

Fig. 1. Behavioral results for sentence reading times and percentage of errors for the sentence comprehension task performed in the scanner. Error bars represent the standard error of the mean.

across-participant averages of the mean integral of percentage change in activation for each of the ROIs, a measure that combines the percent increase in activation over baseline for each significantly activated voxel.

As indicated in Fig. 3, reliably more activation was found in the tongue-twister condition than in the control condition within both the Broca's area ROI (inferior frontal gyrus) $[t(14)=3.08, p<.01]$, an area classically associated with speech production, and the inferior parietal ROI $[t(14)=2.92, p<.05]$. In addition, a trend toward greater activation for the tongue-twister sentences was found in the left superior/middle temporal ROI $[t(14)=1.85, p<.10]$.

Despite relatively large amounts of activation found in the extrastriate (inferior occipital/temporal) and frontal eye fields (precentral sulcus) ROIs, the activation in these areas was not significantly modulated by phonological similarity $[t(14)=0.2$, $p>.5$, and $t(14)=1.36, p>.1$, respectively]. Relatively little activation was found in the left DLPFC (middle frontal gyrus) ROI, and this also was not modulated by phonological similarity $[t(14)=0.83, p>.4]$.

Of the right-hemisphere regions, only the right extrastriate region showed substantial activation. Although there was more activation in the tongue-twister 


\section{Control Sentences}

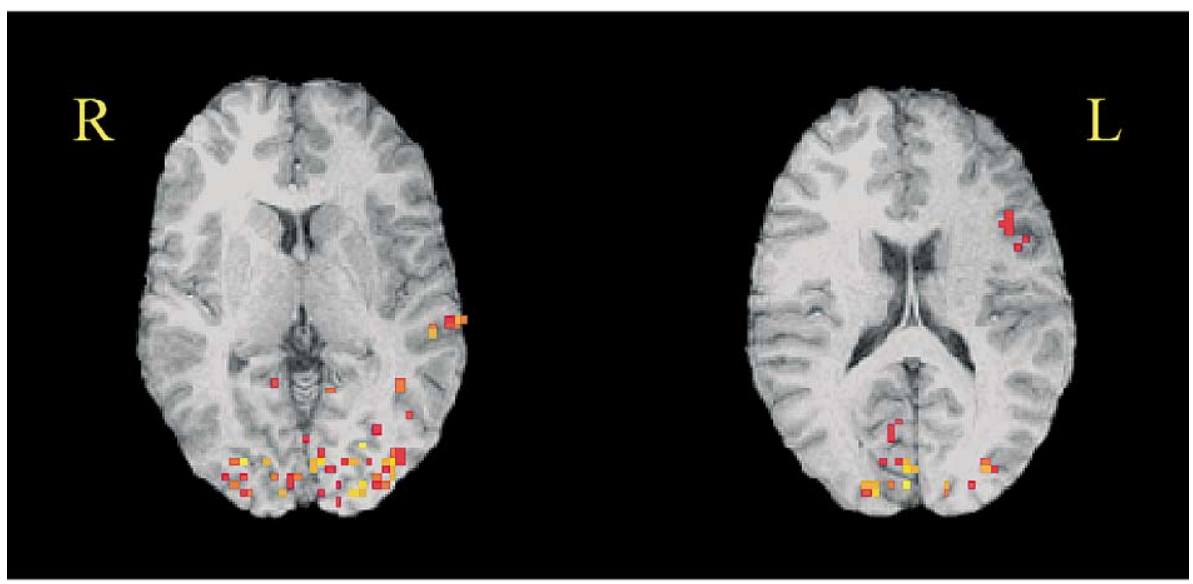

\section{Tongue-twisters}

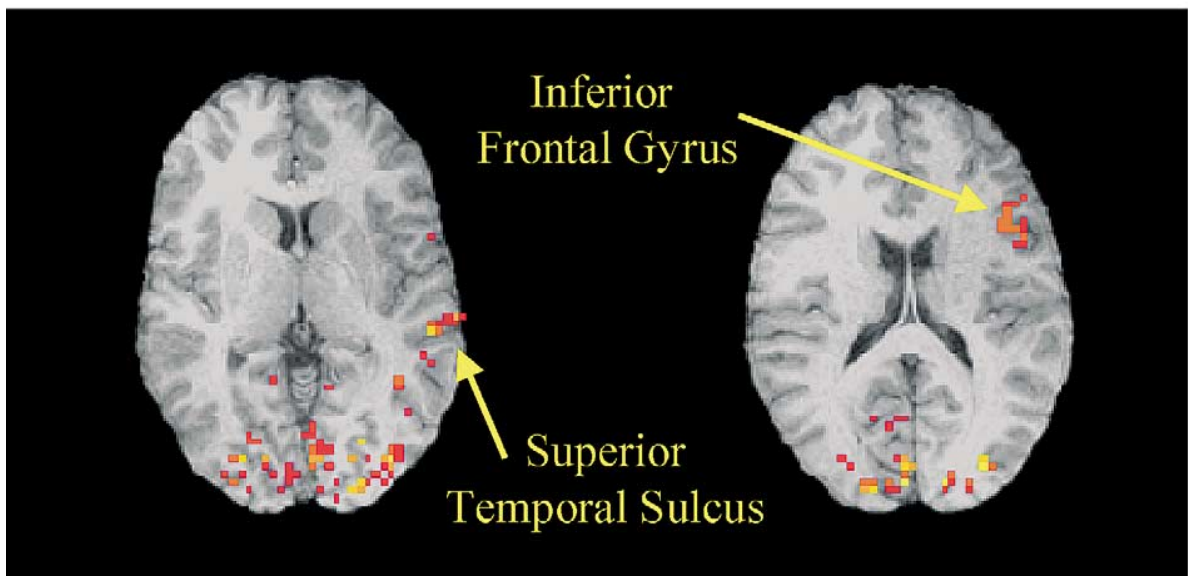

Fig. 2. Functional activation results (thresholded $t$-maps for the comparison between individual conditions and the fixation condition) from a single participant, overlaid on the corresponding structural slices. The slice displayed on the left in each panel is more inferior.

condition, the difference between the two conditions did not reach significance for this ROI $[t(14)=1.70, p>.1]{ }^{1}$ In contrast, both the right inferior frontal ROI and the right frontal eye fields ROI showed relatively little activation across participants, but this activation was nevertheless reliably greater in the tongue-twister conditions $[t(14)=2.47, p<.05$ for the right inferior frontal gyrus, and $t(14)=2.95, p<.05$ for the right frontal eye fields]. In addition there was also a trend toward greater activation for tongue-twisters in the right inferior parietal ROI $[t(14)=1.96, p<.1]$.

There was comparatively little deactivation (voxels showing a reliable decrease in activation relative to the fixation baseline) in the regions of interest examined, and

\footnotetext{
${ }^{1}$ When the data were re-analyzed without first equating for each participant the amount of time spent in the two conditions, the right inferior occipital/temporal extrastriate region was the only area for which the effect of phonological similarity differed from that found in the primary analysis. In this re-analysis, there was reliably more activation $[t(14)=2.42, p<.05]$ in the tongue-twister condition (mean $=25.33$, $\mathrm{SE}=4.49)$ than in the control condition $($ mean $=18.20, \mathrm{SE}=6.31)$.
} 


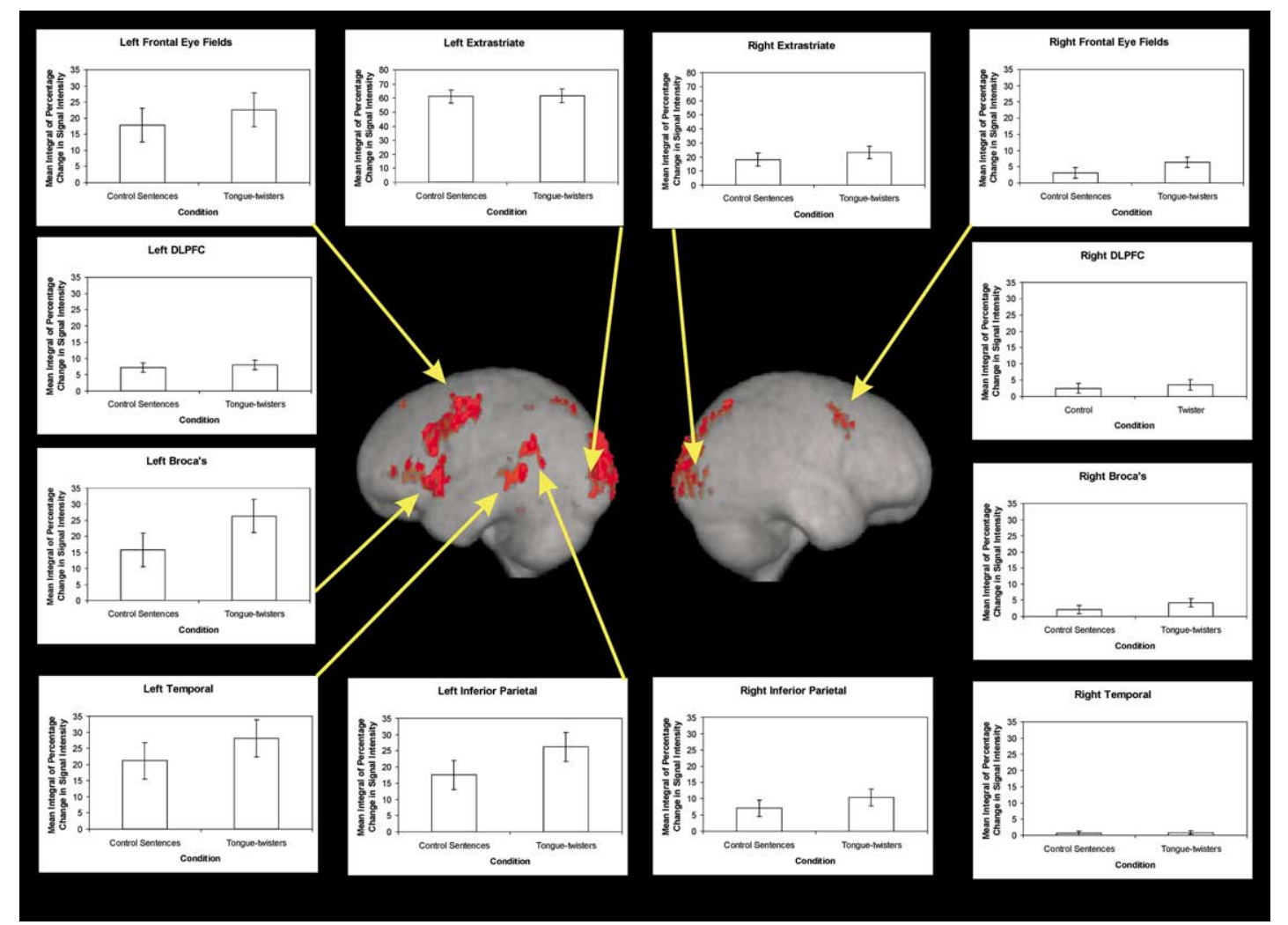

Fig. 3. Plots of the mean integral of the percentage change in signal intensity in each of the ROIs examined. Error bars for the plots represent the standard error of the mean. In the center are group probability maps for the comparison of the tongue-twister condition to the fixation baseline. The probability maps were created by converting each of the 15 participants $t$-maps to $p$-values, transforming the resulting maps into Talairach space, and then using Fisher's method for combining independent $p$-values. The maps were then thresholded to show areas near the cortical surface with a one-tailed $p$-value of $<.001$. These images are intended only to convey common areas of activation across participants in the tongue-twister condition; the statistical analyses of the effect of phonological similarity were carried out upon data from each participant within the individually delineated ROIs. 
more importantly, the deactivation was not reliably modulated by phonological similarity.

Locations of activations within ROIs. The center of activation in each ROI in the two conditions did not differ reliably, suggesting that the two conditions drew on similar networks but simply to different degrees. To assess whether the anatomical location of activation in the two conditions differed systematically across participants, we calculated the differences in the $x, y$, and $z$ coordinates of the centroids of activation between the two conditions for each participant. The mean difference across participants ranged from $0.05 \mathrm{~mm}(\mathrm{SE}=0.33 \mathrm{~mm})$ for the $x$ dimension in the right inferior frontal gyrus $\mathrm{ROI}$ to $3.82 \mathrm{~mm}(\mathrm{SE}=2.29 \mathrm{~mm})$ for the $y$ dimension in the right temporal ROI. None of the mean differences in any dimension or any region was reliably greater than zero following correction for multiple comparisons. Thus, there is no evidence that the additional activation found for the phonologically similar sentences involved a change in the locus of activation in any of the regions examined.

\section{Discussion}

The new neuroimaging results reveal that the added processing load imposed by the silent reading and comprehension of tongue-twister sentences affects a network of areas. The results indicate that both mechanisms hypothesized to be responsible for the tongue-twister effect, working memory processes and articulatory processes, are involved in the phenomenon. Both facets of the neuroimaging results converge well with the expected behavioral finding of slower silent reading of tongue-twister sentences, accompanied by the new behavioral finding of poorer comprehension of tongue-twister sentences.

Internal articulatory-related processes were clearly implicated in the silent reading of tongue-twisters. There was greater activation in the left inferior frontal gyrus (Broca's area, BA 44 and 45) for tongue-twister sentences, explained in terms of more demand placed on the internal articulatory control system. This result suggests that the stimulus sentences were difficult to process because they twist not only the tongue but also the brain. The educated layman's explanation of tongue-twister difficulty is that the actual movements of the articulators are the source of the problem. The additional activation in the left inferior frontal gyrus suggests that tongue-twisters are associated with a corresponding problem in the cortical planning or control or representation of the internal speech. A smaller but reliable effect in the right inferior frontal gyrus is consistent with its involvement in other difficult sentence comprehension tasks (Just et al., 1996). This effect could be pre-lexical or post-lexical.

The neuroimaging results also revealed the effect of tongue-twister reading on other areas associated with the articulatory-phonological loop and internal rehearsal. In addition to the effect found in Broca's area, reliably more activation was found for tongue-twisters than for control sentences in the left angular/supramarginal gyri (BA 39/40), and along the superior temporal sulcus (BA 21/22). These areas have been identified in previous neuroimaging and neuropsychological studies as components of the phonological store in the phonological loop model of working memory (Awh et al., 1996; Fiez et al., 1996; Henson et al., 2000; Honey et al., 2000; Jonides et al., 1998; Paulesu et al., 1993; Vallar et al., 1997; Warrington et al., 1971). A plausible explanation for the finding is that the tongue-twister effect on silent reading times is due to the need to resolve phonological confusions by mentally scanning through a phonological buffer, as suggested by McCutchen et al. (1991). In addition, there was reliably more activation in the tongue-twister condition in a lateral premotor area of the precentral sulcus superior to the inferior frontal sulcus 
(BA 6). Although we refer to this area as the frontal eye fields, it has also been associated with the rehearsal component of the phonological loop (Awh et al., 1996; Henson et al., 2000). In contrast, other brain areas that are not associated with internal articulation or with the articulatory phonological loop were not affected by the experimental manipulation. Regions in the middle frontal gyrus and inferior temporal and ventral occipital extrastriate cortex (BA 18, 19, 37, and 20) did not respond differentially to tongue-twister and control sentences, despite showing robust, leftlateralized responses to the sentences.

The new behavioral evidence of the difficulty of comprehending silently reading tongue-twisters, when juxtaposed with the neuroimaging results, suggests that there may be more reprocessing of the tongue-twister sentences, to overcome the effect of phonological interference (McCutchen \& Perfetti, 1982). Such reprocessing could conceivably have involved the adoption of a strategy of overtly re-reading the visually presented sentences in the tongue-twister condition. If this overt re-reading interpretation were correct, one might expect greater activation for the tongue-twister sentences in early visual areas of the occipital and temporal lobes, particularly in the left hemisphere, given previous findings that the extrastriate response during reading is left lateralized (Keller et al., 2001). However, contrary to this expectation, there was no reliable difference for the left extrastriate region in the present study. As the technological capability to obtain neuroimaging and eye-tracking measures simultaneously becomes more available, such hypotheses will be testable even more directly. The behavioral data demonstrated a tongue-twister effect not only on the time it takes to silently read sentences, but also on comprehension performance. Although previous behavioral studies have shown that it is more difficult to make semantic acceptability judgments on tongue-twister sentences (Hanson et al., 1991; McCutchen \& Perfetti, 1982; Zhang \& Perfetti, 1993), it could be argued that such a task involves processes and strategies that are not like those used in normal reading or are less demanding than those used in normal reading. The present study extends the generality of the visual tongue-twister effect on comprehension performance to a more natural reading situation. The behavioral findings dovetail with the brain imaging findings in providing a more detailed characterization of the tongue-twister effect.

\section{Acknowledgments}

This research was supported in part by the National Institute for Neurological Disorders and Stroke, Grant P01NS-35949, the National Institute of Mental Health Grant MH-29617, the National Institute of Mental Health, Senior Scientist Awards, MH-00661 and MH-00662. We thank Jennifer K. Roth for assistance in the design and analysis of the experiment and Chuck Perfetti for helpful comments on a previous draft.

\section{References}

Awh, E., Jonides, J., Smith, E. E., Schumacher, E. H., Koeppe, R. A., \& Katz, S. (1996). Dissociation of storage and rehearsal in verbal working memory: Evidence from positron emission tomography. Psychological Science, 7, 25-31.

Ayres, T. J. (1984). Silent reading time for tongue-twister paragraphs. American Journal of Psychology, 97, 605-609.

Baddeley, A. D., \& Hitch, G. (1974). Working memory. In G. H. Bower (Ed.), The psychology of learning and motivation (8, pp. 47-89). New York: Academic Press. 
Baddeley, A. D., \& Lewis, V. J. (1981). Inner active processes in reading: The inner ear, inner voice, and inner eye. In A. M. Lesgold \& C. A. Perfetti (Eds.), Interactive processes in reading (pp. 299-327). Hillsdale, NJ: Erlbaum.

Baddeley, A. D., Thomson, N., \& Buchanan, M. (1975). Word length and the structure of short-term memory. Journal of Verbal Learning and Verbal Behavior, 14, 575-589.

Braver, T., Cohen, J. D., Jonides, J., Smith, E. E., \& Noll, D. C. (1997). A parametric study of prefrontal cortex involvement in human working memory. NeuroImage, 5, 49-62.

Carpenter, P. A., Just, M. A., Keller, T., Eddy, W. F., \& Thulborn, K. R. (1999). Graded functional activation in the visuospatial system with the amount of task demand. Journal of Cognitive Neuroscience, 11, 9-24.

Caviness, V. S., Jr., Meyer, J., Makris, N., \& Kennedy, D. N. (1996). MRI-based topographic parcellation of human neocortex: An anatomically specified method with estimate of reliability. Journal of Cognitive Neuroscience, 8, 566-587.

Chee, M. W., Tan, E. W., \& Thiel, T. (1999). Mandarin and English single word processing studied with functional magnetic resonance imaging. Journal of Neuroscience, 19, 3050-3056.

Coltheart, M., Brent, C., Atkins, P., \& Haller, M. (1993). Models of reading aloud: Dual-route and parallel-distributed-processing approaches. Psychological Review, 100, 589-608.

Conrad, R. (1964). Acoustic confusions in immediate memory. British Journal of Psychology, 55, 75-84.

Cuenod, C. A., Bookheimer, S. Y., Hertz-Pannier, L., Zeffiro, T. A., Theodore, W. H., \& Le Bihan, D. (1995). Functional MRI during word generation, using conventional equipment: A potential tool for language localization in the clinical environment. Neurology, 45, 1821-1827.

Demonet, J.-F., Chollet, F., Ramsay, S., Cardebat, D., Nespoulous, J.-L., Wise, R., Rascol, A., \& Frackowiak, R. (1992). The anatomy of phonological and semantic processing in normal subjects. Brain, 115, 1753-1768.

Eddy, W. F., Fitzgerald, M., Genovese, C. R., Mockus, A., \& Noll, D. C. (1996). Functional imaging analysis software computational olio. In Proceedings in computational statistics (pp. 39-49). Heidelberg: Physica-Verlag.

Fiez, J. A., Raife, E. A., Balota, D. A., Schwarz, J. P., Raichle, M. E., \& Petersen, S. E. (1996). A positron emission tomography study of the short-term maintenance of verbal information. Journal of Neuroscience, 16, 808-822.

Frith, C. D., Friston, K. J., Liddle, P. F., \& Frackowiak, R. S. J. (1991). A PET study of word finding. Neuropsychologia, 29, 1137-1148.

Gathercole, S. E., \& Baddeley, A. D. (1993). Working memory and language. Hillsdale, NJ: Erlbaum.

Grasby, P. M., Frith, C. D., Friston, K. J., Simpson, J., Fletcher, P. C., Frackowiak, R. S. J., \& Dolan, R. J. (1994). A graded task approach to the functional mapping of brain areas implicated in auditoryverbal memory. Brain, 117, 1271-1282.

Haber, L. R., \& Haber, R. N. (1982). Does silent reading involve articulation. Evidence from tonguetwisters. American Journal of Psychology, 95, 410-419.

Hanson, V. L., Goodell, E. W., \& Perfetti, C. A. (1991). Tongue-twister effects in the silent reading of hearing and deaf college students. Journal of Memory and Language, 30, 319-330.

Henson, R. N. A., Burgess, N., \& Frith, C. D. (2000). Recoding, storage rehearsal, and grouping in verbal short-term memory: An fMRI study. Neuropsychologia, 38, 426-440.

Hinke, R. M., Hu, X., Stillman, A. E., Kim, S.-G., Merkle, H., Salmi, R., \& Ugurbil, K. (1993). Functional magnetic resonance imaging of Broca's area during internal speech. NeuroReport, 4, 675-678.

Honey, G. D., Bullmore, E. T., \& Sharma, T. (2000). Prolonged reaction time to a verbal working memory task predicts increased power of posterior parietal cortical activation. NeuroImage, 12, 495-503.

Johnston, R. S., Thompson, G. B., Gletcher-Flinn, C. M., \& Holligan, C. (1995). The functions of phonology in the acquisition of reading: Lexical and sentence processing. Memory and Cognition, 23, 749-766.

Jonides, J., Schumacher, E. H., Smith, E. E., Koeppe, R. A., Awh, E., Reurter-Lorenz, P. A., Marshuetz, C., \& Willis, C. R. (1998). The role of the parietal cortex in verbal working memory. Journal of Neuroscience, 18, 5026-5034.

Just, M. A., Carpenter, P. A., Keller, T. A., Eddy, W. F., \& Thulborn, K. R. (1996). Brain activation modulated by sentence comprehension. Science, 274, 114-116.

Keller, T. A., Carpenter, P. A., \& Just, M. A. (2001). The neural bases of sentence comprehension: A fMRI examination of syntactic and lexical processing. Cerebral Cortex, 11, 223-237.

Kucera, F., \& Francis, W. N. (1967). Computational analysis of present-day American English. Providence, RI: Brown University Press.

Lukatela, G., \& Turvey, M. T. (1990). Phonemic similarity effects and prelexical phonology. Memory and Cognition, 18, 128-152.

Lukatela, G., \& Turvey, M. T. (1994a). Visual lexical access is initially phonological: 1. Evidence from associative priming by words, homophones, and pseudohomophones. Journal of Experimental Psychology: General, 123, 107-128. 
Lukatela, G., \& Turvey, M. T. (1994b). Visual lexical access is initially phonolgical: 2. Evidence from phonological priming by homophones and pseudohomophones. Journal of Experimental Psychology: General, 123, 331-353.

McCutchen, D., Bell, L. C., France, I. M., \& Perfetti, C. A. (1991). Phoneme-specific interference in reading: The tongue-twister effect revisited. Reading Research Quarterly, 26, 87-103.

McCutchen, D., Dibble, E., \& Blount, M. M. (1994). Phonemic effects in reading comprehension and text memory. Applied Cognitive Psychology, 8, 597-611.

McCutchen, D., \& Perfetti, C. A. (1982). The visual tongue-twister effect: Phonemic activation in silent reading. Journal of Verbal Learning and Verbal Behavior, 21, 672-687.

Naish, P. (1980). The effects of graphemic and phonemic similarity between targets and masks in a backward visual masking paradigm. Quarterly Journal of Experimental Psychology, 32, 57-68.

Oldfield, R. C. (1971). The assessment and analysis of handedness: The Edinburgh inventory. Neuropsychologia, 87, 256-259.

Paulesu, E., Frith, C. D., \& Frackowiak, R. S. J. (1993). The neural correlates of the verbal component of working memory. Nature, 362, 342-345.

Plaut, D. C., McClelland, J. L., Seidenberg, M. S., \& Patterson, K. (1996). Understanding normal and impaired word reading: Computational principles in quasi-regular domains. Psychological Review, 103, 56-115.

Postma, A., \& Noordanus, C. (1996). Production and detection of speech errors in silent, mouthed, noisemasked, and normal auditory feedback speech. Language and Speech, 39, 375-392.

Rademacher, J., Galaburda, A. M., Kennedy, D. N., Filipek, P. A., \& Caviness, V. S., Jr. (1992). Human cerebral cortex: Localization, parcellation, and morphometry with magnetic resonance imaging. Journal of Cognitive Neuroscience, 4, 352-374.

Robinson, D. H., \& Katayama, A. D. (1997). At-lexical, articulatory interference in silent reading: The "upstream" tongue-twister effect. Memory and Cognition, 25, 661-665.

Rosenbaum, D. A., Weber, R. J., Hazelett, W. M., \& Hindorff, V. (1986). The parameter remapping effect in human performance: Evidence from tongue twisters and finger fumblers. Journal of Memory and Language, 25, 710-725.

Rypma, B., Prabhakaran, V., Desmond, J. E., Glover, G. H., \& Gabrieli, J. D. E. (1999). Load-dependent roles of frontal brain regions in the maintenance of working memory. NeuroImage, 9, 216-226.

Seidenberg, M. S., \& McClelland, J. L. (1989). A distributed, developmental model of word recognition and naming. Psychological Review, 96, 523-568.

Seidenberg, M. S., Waters, G. S., Barnes, M. A., \& Tanenhaus, M. K. (1984). When does irregular spelling or pronunciation influence word recognition? Journal of Verbal Learning and Verbal Behavior, 23, 383-404.

Thulborn, K. R., Voyvodic, J., McCurtain, B., Gillen, J., Chang, S., Just, M. A., Carpenter, P. A., \& Sweeney, J. A. (1996). High field functional MRI in humans: Applications to cognitive function. In P. Pavolone \& P. Rossi (Eds.), Proceedings of the European seminars in diagnostic and interventional imaging, Syllabus 4 (pp. 91-96). New York: Springer-Verlag.

Vallar, G., \& Cappa, S. F. (1987). Articulation and verbal short-term memory: Evidence from anarthria Cognitive Neuropsychology, 4, 55-78.

Vallar, G., Di Betta, A. M., \& Silveri, M. C. (1997). The phonological short-term store-rehearsal system: Patterns of impairment and neural correlates. Neuropsychologia, 35, 795-812.

Van Orden, G. C., Pennington, B. F., \& Stoner, G. O. (1990). Word identification in reading and the promise of subsymbolic psycholinguistics. Psychological Review, 97, 488-522.

Van Orden, G. D. (1987). A ROWS is a ROSE: Spelling, sound, and reading. Memory and Cognition, 15, 181-198.

Wagner, R. K., \& Torgeson, J. K. (1987). The nature of phonological processing and its causal role in the acquisition of reading skills. Psychological Bulletin, 101, 192-212.

Warrington, E. K., Logue, V., \& Pratt, R. T. C. (1971). The anatomical localization of selective impairment of auditory verbal short-term memory. Neuropsychologia, 9, 37-387.

Waters, G. S., \& Seidenberg, M. S. (1985). Spelling-sound effects in reading: Time-course and decision criteria. Memory and Cognition, 13, 557-572.

Waters, G. S., Seidenberg, M. S., \& Bruck, M. (1984). Children's and adults' use of spelling sound information in three reading tasks. Memory and Cognition, 12, 293-305.

Wise, R., Chollet, F., Hadar, U., Friston, K., Hoffner, E., \& Frackowiak, R. (1991). Distribution of cortical neural networks involved in word comprehension and word retrieval. Brain, 114, 1803-1817.

Xiong, J., Rao, S., Gao, J.-H., Woldorff, M., \& Fox, P. T. (1998). Evaluation of hemispheric dominance for language using functional MRI: A comparison with positron emission tomography. Human Brain Mapping, 6, 42-58.

Zhang, S., \& Perfetti, C. A. (1993). The tongue-twister effect in reading Chinese. Journal of Experimental Psychology: Learning, Memory, and Cognition, 19, 1082-1093. 